\title{
SENSING LIQUID PROPERTIES WITH SHEAR-MODE RESONATOR SENSORS
}

\author{
S. J. Martin*; R. W. Cernosek*, and J. J. Spates** \\ *Sandia National Laboratories, Albuquerque, NM, 87185 U.S.A. \\ **Ktech Corporation, Albuquerque, NM 87110 U.S.A.
}

\section{SUMMARY}

Liquid properties are measured from the changes they induce in the resonant frequency and damping of thicknessshear mode quartz resonators. A smooth-surfaced resonator viscously entrains the contacting fluid and responds to the density-viscosity product. Separation of density and viscosity is accomplished using two devices: one with a smooth surface and one with a corrugated surface that traps fluid. By observing the difference in stored and dissipated energies in the contacting fluid, its non-Newtonian characteristics can also be determined.

\section{INTRODUCTION}

Piezoelectric devices that can be electrically excited into a shear mode of vibration can be used to measure fluid properties. These devices typically consist of a thin disk of AT-cut quartz with electrodes patterned on both sides (Fig. 1). When excited at resonance, the crystal vibrates in a thickness-shear mode (TSM) with faces undergoing inplane displacement. When operated in a fluid, the in-plane surface oscillation radiates a critically-damped shear wave into the contacting fluid (Fig. 1). The decay length of this wave is $\delta=\left(2 \eta /\left(\omega_{s} \rho\right)\right)^{1 / 2}[1]$, where $\eta$ and $\rho$ are the fluid viscosity and density and $\omega_{s}=2 \pi f_{s}$, where $f_{s}$ is the series resonant frequency of the unperturbed (dry) device.

\section{EQUIVALENT-CIRCUIT MODEL}

The mechanical interaction between the piezoelectric crystal and the contacting fluid results in an electrical response that indicates fluid properties. Fig. 2 shows an

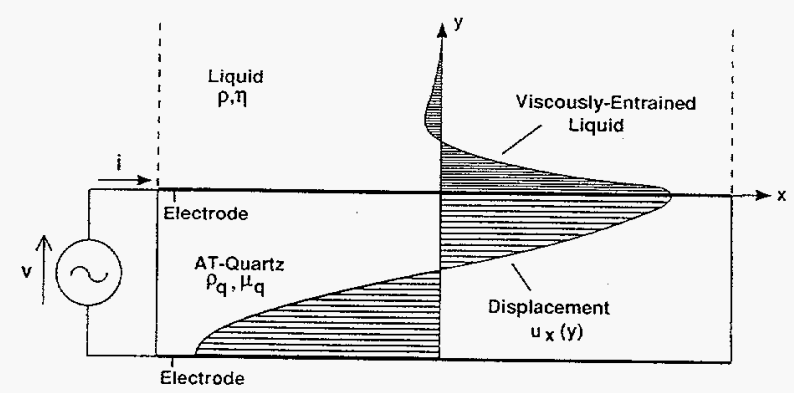

Fig. 1. Cross-sectional view of a smooth TSM resonator with the upper surface contacted by a liquid. Shear motion of the smooth surface causes a thin layer of the contacting liquid to be viscously entrained. equivalent-circuit model that describes the electrical characteristics of the TSM resonator with a contacting fluid [2]. This model consists of a "static" capacitance $C_{0}{ }^{*}$ (crystal device capacitance $C_{0}$ plus external parasitic capacitance $\left.C_{p}\right)$ in parallel with a "motional branch" $\left(L_{1}, C_{1}\right.$, $\left.R_{1}, L_{2}, R_{2}, L_{3}\right)$. The motional branch arises from electrical excitation of a shear-mode mechanical resonance in the piezoelectric quartz. This branch gives rise to a series resonant frequency $f_{s}=1 /\left(2 \pi\left(L C_{1}\right)^{1 / 2}\right)$, where $L$ is the total motional inductance.

The motional impedance is increased by the mechanical interaction between the resonator and a contacting fluid. The energy stored and dissipated in the fluid are represented by a motional inductance $L_{2}$ and resistance $R_{2}$ and are shown in Fig. 2. These elements are related to properties of the resonator and the contacting (Newtonian) fluid by $[2,3]$ :

$$
R_{2}=X_{2}=\omega_{s} L_{2}=\frac{N \pi}{4 K^{2} C_{o}}\left(\frac{\rho \eta}{2 \omega_{s} \mu_{q} \rho_{q}}\right)^{\frac{1}{2}}
$$

where $\mathrm{K}^{2}, \mu_{\mathrm{q}}$ and $\rho_{\mathrm{q}}$ are the electromechanical coupling coefficient, shear stiffness, and density of the quartz; $N$ is the harmonic number. We note that for Newtonian fluids,

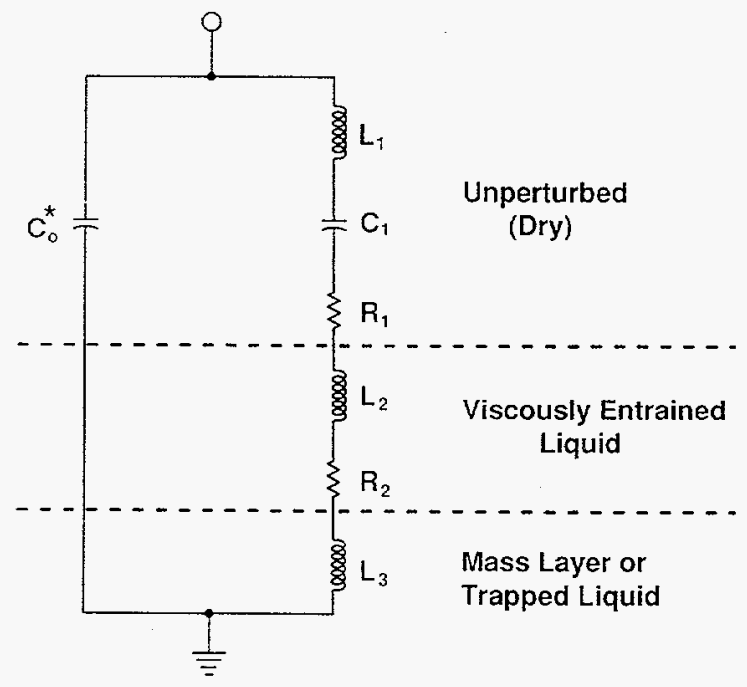

Fig. 2. Equivalent-circuit model used to describe the electrical characteristics of a TSM resonator with liquid loading. Contributions arise from both viscously-entrained liquid and liquid trapped by surface asperities. 


\section{DISCLAIMER}

This report was prepared as an account of work sponsored by an agency of the United States Government. Neither the United States Government nor any agency thereof, nor any of their employees, makes any warranty, express or implied, or assumes any legal liability or responsibility for the accuracy, completeness, or usefulness of any information, apparatus, product, or process disclosed, or represents that its use would not infringe privately owned rights. Reference herein to any specific commercial product, process, or service by trade name, trademark, manufacturer, or otherwise does not necessarily constitute or imply its endorsement, recommendation, or favoring by the United States Government or any agency thereof. The views and opinions of authors expressed herein do not necessarily state or reflect those of the United States Government or any agency thereof. 


\section{DISCLAIMER}

Portions of this document may be illegible in electronic image products. Images are produced from the best available original document. 
$R_{2} \equiv X_{2}$ and depends on fluid properties as $(\rho \eta)^{1 / 2}$.

The equivalent-circuit model of Fig. 2 also has a motional contribution $L_{3}$ arising from a mass layer or "trapped" liquid. Liquid can be constrained by closelyspaced vertical surface features to move synchronously with the device surface [3,4], rather than undergoing a progressive phase lag with distance from the surface as occurs with a viscously-entrained fluid.

\section{LIQUID-LOADING RESPONSES}

Fig. 3 shows the electrical admittance vs. excitation frequency measured (points) for several glycerol solutions contacting one side of a TSM resonator. (Admittance is the ratio of current $i$ to applied voltage $v$ shown in Fig. 1.) Both admittance magnitude and phase were measured with a network analyzer. The dashed line in Fig. 3 indicates the series resonant frequency $\left(f_{s}\right)$ for the dry device. As $\rho \eta$ increases with glycerol concentration (in water), the plot of admittance magnitude shows both a translation of the series resonance peak and a diminution and broadening of the peak. The solid lines in Fig. 3 are calculated from the equivalent-circuit model of Fig. 2 using a best-fit value of $\rho \eta$ along with values of $C_{0}, L_{1}, C_{1}$, and $R_{1}$ obtained by fitting measurements made on the dry device.

Resonator responses are typically reported in terms of changes in resonant frequency, $\Delta f$, and motional resistance, $\Delta R$ (equal to $R_{2}$ ). The equivalent-circuit model can be used to predict the shift in series resonant frequency with liquid properties [2,3]:

$$
\Delta f \cong-\frac{\left(L_{2}+L_{3}\right) f_{s}}{2 L_{1}}=-\frac{2 f_{s}^{2}}{N\left(\mu_{q} \rho_{q}\right)^{1 / 2}}\left[\left(\frac{\rho \eta}{4 \pi f_{s}}\right)^{\frac{1}{2}}+\rho h\right]
$$

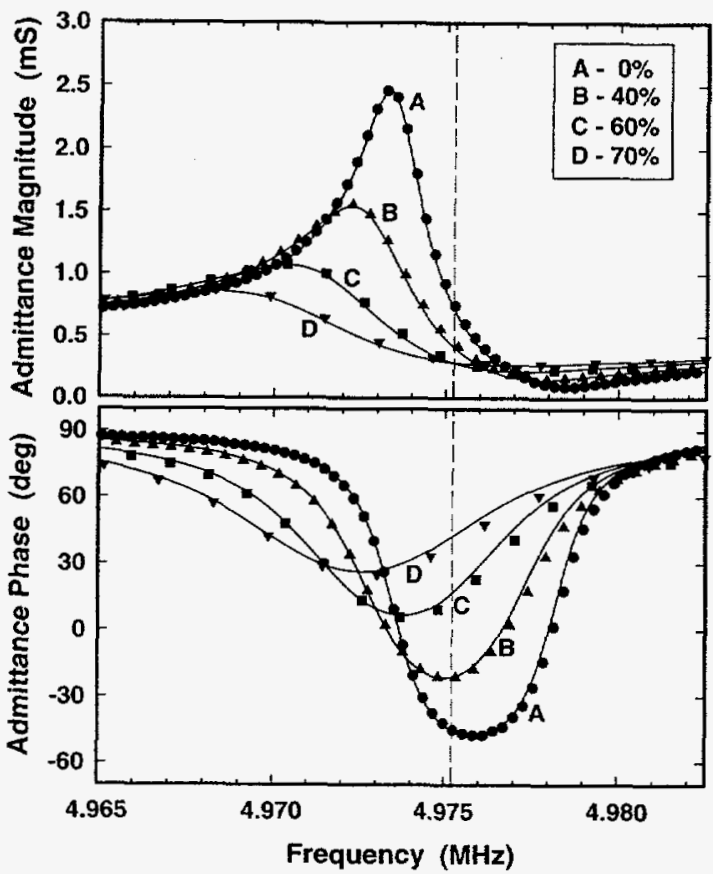

Fig. 3. Resonator electrical admittance measured (points) and calculated (ines) vs. frequency for several glycerol concentrations (wt. \%) in water.
The first term of Eq. 2 predicts a linear decrease in resonant frequency with $(\rho \eta)^{1 / 2}$, in agreement with Kanazawa and Gordon [1]. The second term is an offset that arises from a mass layer or trapped fluid layer having density $\rho$ and effective thickness $h$.

Fig. 4 shows the changes in series resonant frequency $\Delta f$ and motional resistance $\Delta R$ (from the data of Fig. 3) vs. the liquid parameter $(\rho \eta)^{1 / 2}$. Two different resonators were examined: a hydrodynamically smooth device with average surface roughness less than $10 \mathrm{~nm}$ and a "rough" device with average roughness of approximately $243 \mathrm{~nm}$. The liquid responses, $\Delta \mathrm{f}$ and $\Delta R$ vary linearly with $(\rho \eta)^{1 / 2}$; the dashed lines are calculated from Eqs. 1 and 2 assuming an ideally smooth surface $(h=0)$. The smooth device, with roughness features much smaller than the decay length $\delta(250 \mathrm{~nm}$ in water at $5 \mathrm{MHz}$ ), exhibits responses close to the smoothsurface predictions. The rough device, however, exhibits a significant offset in frequency (and slightly greater slope) due to liquid trapping in surface features. The offset indicates an effective thickness $h \cong 150 \mathrm{~nm}$--about $62 \%$ of the measured surface roughness.

\section{OSCILLATOR CIRCUIT}

A "lever oscillator" circuit has been developed to operate TSM resonators in contact with fluids [5]. This oscillator (Fig. 5) uses feedback to servo the impedance phase to near zero, ensuring that the oscillation frequency is approximately $f_{s^{\circ}}$ Additionally, the circuit has an automatic level control (ALC) to vary the loop gain to compensate for crystal damping induced by liquid loading. This circuit will sustain oscillation in relatively viscous media $(\eta \leq 200 \mathrm{cP})$. The loop feedback voltage is proportional to $R_{2}$ and hence

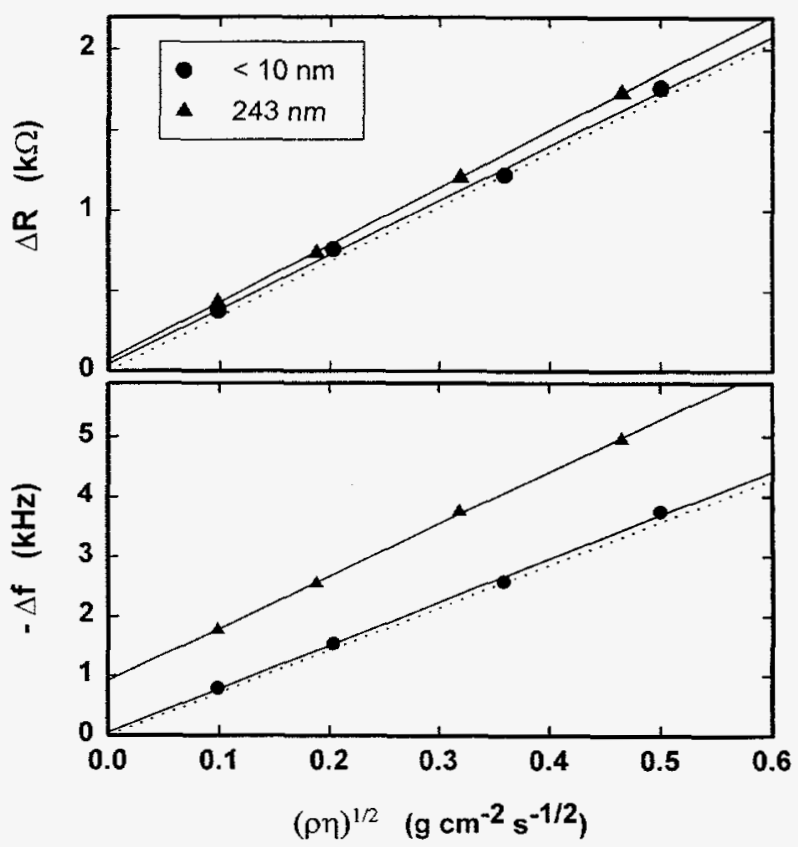

Fig. 4. Change in series resonant frequency, $\Delta f$, and motional resistance, $\Delta R$, vs. the liquid parameter $(\rho \eta)^{1 / 2}$ for TSM resonators with two different surface roughnesses. 
$(\rho \eta)^{1 / 2}$. This feedback voltage is taken as an output to indicate fluid properties. While a surface mass layer or trapped liquid layer will affect the oscillation frequency, the motional resistance and feedback voltage are unaffected $[6,7]$.

The response of a resonator/oscillator combination to liquid loading may differ from that of the resonator alone. Fig. 6 shows such variations in resonant frequency as a function of the liquid parameter $(\rho \eta)^{1 / 2}$. The resonator's series resonant frequency, measured with a network ana-

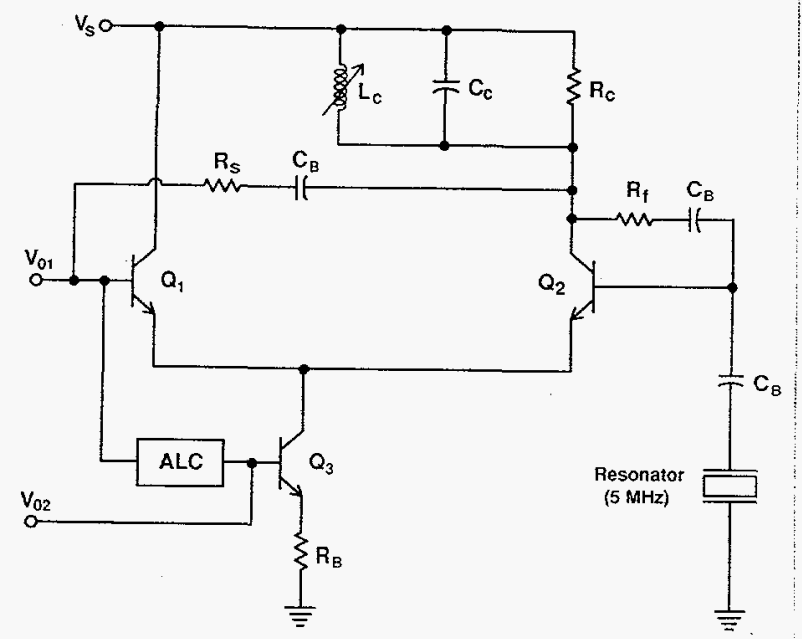

Fig. 5. Oscillator circuit designed to operate the TSM resonator in liquid media, providing two outputs: $V_{o 1}(R F$ output) indicates oscillation frequency; $V_{O 2}$ (DC output) is proportional to motional resistance.

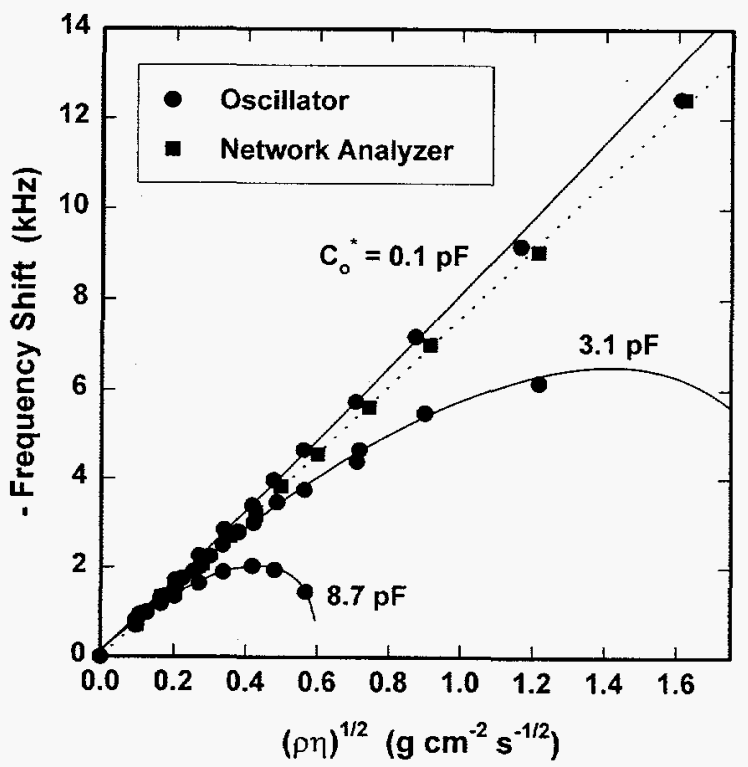

Fig. 6. Frequency shift vs. the liquid loading parameter $(\rho \eta)^{1 / 2}$ for (a) the series resonant frequency measured with a network analyzer ( $\mathbf{0})$, and (b) the resonator/oscillator combination measured for several values of the static capacitance $C_{o}{ }^{*}(\bullet)$. lyzer, varies linearly with $(\rho \eta)^{1 / 2}$, according to Eq. 2 . The resonator/oscillator response to liquid loading, however, depends upon the capacitance $C_{o}^{*}$. For the untuned device, $C_{o}^{*}=8.7 \mathrm{pF}$. As this capacitance is tuned out, using an inductor in parallel with the resonator, the frequency shift is controlled more by the resonator and less by the oscillator. Moreover, decreasing $C_{o}^{*}$ increases the range of liquid properties over which the oscillator will function.

\section{SEPARATION OF DENSITY AND VISCOSITY}

Fluid density and viscosity can be resolved by using a pair of smooth and corrugated resonators (Fig. 7 inset) [8]. The smooth resonator response depends only on $\rho \eta$; the corrugated resonator exhibits an additional frequency shift (Eq. 2, second term) proportional to $\rho$, due to liquid trapping in the surface features. Thus, the responses from the resonator combination resolves density and viscosity. Fig. 7 shows density and viscosity for a number of fluids (Table I) extracted from measurements made with the combination of smooth and corrugated resonators. The surface corrugation consists of ridges $5 \mu \mathrm{m}$ wide and $1.5 \mu \mathrm{m}$ high, formed by patterned gold electroplating.

Table I. Liquids shown in Fig. 7.

\begin{tabular}{|c|l||c|l|}
\hline A & n-pentane & H & n-hexanol \\
\hline B & n-hexane & I & n-heptanol \\
\hline C & methanol & J & n-octanol \\
\hline D & ethanol & K & dichloromethane \\
\hline E & n-propanol & L & trichloroethylene \\
\hline F & n-butanol & M & carbon tetrachloride \\
\hline G & n-pentanol & N & dibromomethane \\
\hline
\end{tabular}

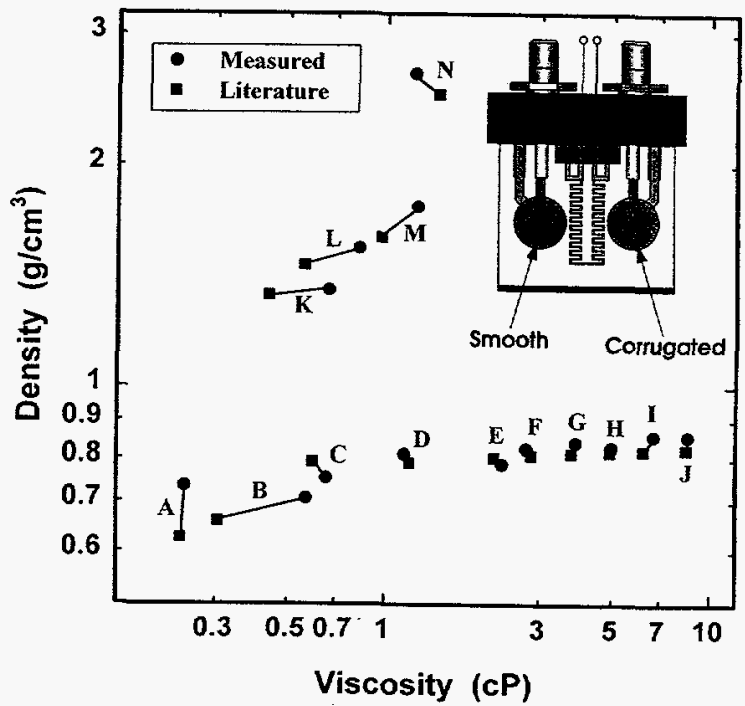

Fig. 7. Density and viscosity extracted using the corrugated and smooth resonator pair (inset) (৫) along with literature values (ᄈ) for liquids in Table I. 


\section{NON-NEWTONIAN FLUIDS}

Eq. 1, derived for loading by a Newtonian fluid, gives the relation $X_{2} \otimes R_{2}$. For more complicated fluids, this relationship breaks down. We consider the Maxwell fluid, for example, whose viscosity exhibits a relaxation response with frequency [9]: $\eta(\omega)=\eta_{0} /(1+j \omega \tau)$, where $\eta_{0}$ is the low-frequency viscosity and $\tau$ is the fluid relaxation time defined by $\tau=\eta_{0} / \mu$, where $\mu$ is the high frequency shear modulus of the fluid. The Maxwell fluid reduces to a Newtonian fluid when $\omega \tau$ « 1 . The motional impedance elements arising from Maxwell-fluid loading are:

$$
\begin{aligned}
& X_{2}=X_{2}^{(N)}\left(1-\frac{\omega \tau}{\sqrt{1+(\omega \tau)^{2}}}\right) \\
& R_{2}=R_{2}^{(N)}\left(1+\frac{\omega \tau}{\sqrt{1+(\omega \tau)^{2}}}\right)
\end{aligned}
$$

where $X_{2}^{(N)}$ and $R_{2}^{(N)}$ denote the motional impedance elements for a Newtonian fluid (Eq. 1): $X_{2}^{(N)} \equiv R_{2}^{(N)}$. Note from Eqs. 3 that $R_{2}=X_{2}$ only for a Newtonian fluid ( $\left.\omega \tau \ll 1\right)$; as $\omega \tau$ approaches 1 , the second term in Eqs. 3 becomes appreciable so that $R_{2}>X_{2}$. This serves as an indicator for non-Newtonian fluid behavior.

Fig. 8 shows $X_{2}$ and $R_{2}$ extracted from network analyzer measurements made on resonators immersed in two synthetic motor oils: a synthetic lubricant base stock and the fully-formulated lubricant with polymeric viscosity index improvers. At high temperature $R_{2}=X_{2}$ for both lubricants, indicating Newtonian fluid behavior. At low temperature, we find $R_{2}$ diverges from $X_{2}$, with $R_{2}>X_{2}$. These results are

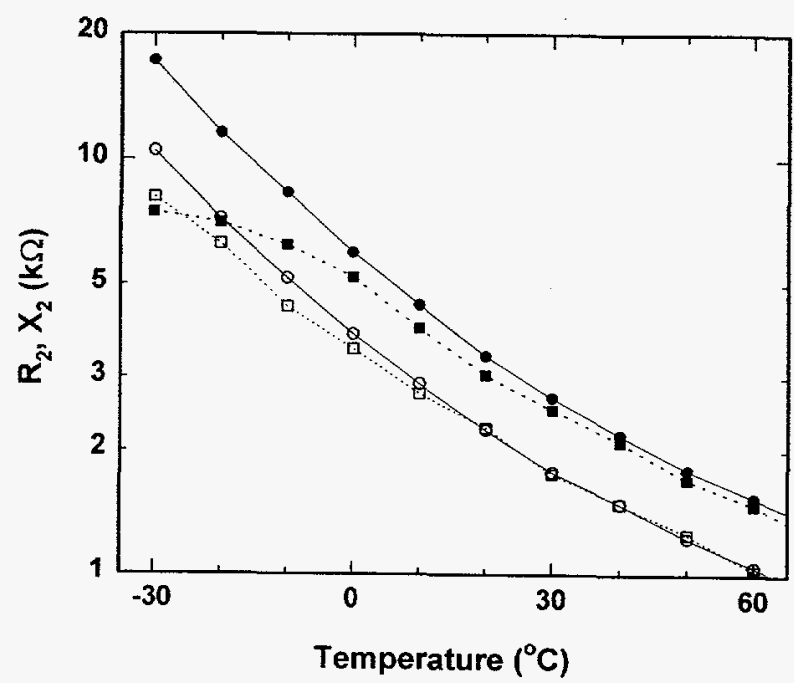

Fig. 8. Motional resistance $R_{2}$ and reactance $X_{2}$ vs. temperature for two synthetic lubricants: $R_{2}(O)$ and $X_{2}(\square)$ for the synthetic base stock; $R_{2}(\bullet)$ and $X_{2}(\square)$ for the fully-formulated lubricant with polymeric additives. consistent with the Maxwell fluid model if we note that $\eta_{\mathrm{o}}$ varies inversely with temperature, while $\mu$ is comparatively temperature independent. At high temperatures, $\eta_{0}$ is low, resulting in $\omega \tau=\omega \eta_{0} / \mu<1$ and Newtonian fluid behavior; at low temperatures, $\eta_{0}$ is high and $\omega \tau-1$, causing nonNewtonian behavior. In Fig. 8, the non-Newtonian effects are more pronounced for the fully-formulated lubricant due to the addition of polymeric additives; these large molecules increase relaxation time $\tau$.

\section{CONCLUSION}

Shear-mode resonators can be used to measure the density and viscosity of contacting fluids. Additionally, fluid degradation and non-Newtonian behavior, both related to changes in viscous properties, can also be determined. These devices are ideal for many sensing applications: they are small and inexpensive, very robust (can operate in high temperature, high vibration, and highly corrosive environments), have no macroscopic moving parts, and can be configured to function as in situ fluid monitors.

\section{ACKNOWLEDGEMENTS}

The authors are grateful for assistance from K. O. Wessendorf, T. W. Schneider, G. C. Frye, M. A. Mitchell, A. N. Rumpf, and L. Casaus of Sandia National Laboratories, S. D. Senturia of the Massachusetts Institute of Technology, and R. J. Huber of the University of Utah. This work was performed at Sandia National Laboratories, supported by the U.S. Department of Energy under contract No. DEAC04-94AL85000.

\section{REFERENCES}

[1] Kanazawa, K. K.; Gordon II, J. G. Anal. Chem. 1985, 57, 1770-1771.

[2] Martin, S. J.; Granstaff, V. E.; Frye, G. C. Anal. Chem. 1991, 63, 2272-2281.

[3] Martin, S. J.; Frye, G. C.; Ricco, A. J.; Senturia, S. D. Anal. Chem. 1993, 65, 2910-2922.

[4] Schumacher, R. Angew. Chem., Int. Ed. Engl. 1990, 61, 290-295.

[5] Wessendorf, K. O. Proc. 1993 Frequency Control Symp. (IEEE, New York, 1993) pp. 711-717.

[6] Martin, S. J.; Frye, G. C. Proc. 1991 Ultrasonics Symposium, (IEEE, New York, 1991), 393-398.

[7] Mecea, V. M. Sensors and Actuators A 1994, 40, 127.

[8] Martin, S. J.; Frye, G. C.; Wessendorf, K. O. Sensors and Actuators A 1994, 44, 209-218.

[9] White, F. M. Viscous Fluid Flow, McGraw-Hill: New York, 1991, Sec. 3-5.1. 